Mathematische Semesterberichte manuscript No.

(will be inserted by the editor)

Michael Breuß . Andreas Meister .

Thomas Sonar

\title{
Modellierung und numerische Simulation der Thermoregulation von Früh- und Neugeborenen
}

Eingegangen: date / Angenommen: date

Zusammenfassung Bei frühgeborenen Säuglingen spielt die Thermoregulation zur Aufrechterhaltung einer überlebenswichtigen Körpertemperatur durch Wärmeproduktion, -abgabe bzw. -aufnahme eine entscheidende Rolle. Der Einsatz moderner Inkubatoren soll die körpereigenen Thermoregulatoren unterstützen, und es ist im Hinblick auf verschiedene medizinische Fragestellungen wünschenswert, diesen Prozess modellieren zu können. Wir stellen

M. Breuß

TU Braunschweig

Carl-Friedrich-Gauß-Fakultät für Mathematik und Informatik

Computational Mathematics

Pockelsstraße 14

D-38106 Braunschweig

Tel.: +531-391-7419

Fax: +531-391-7409

E-mail: m.breuss@tu-bs.de

A. Meister

Fachbereich für Mathematik und Informatik

AG Analysis und Angewandte Mathematik

Universität Kassel

Heinrich Plett Str. 40 (AVZ)

D-34132 Kassel

Tel.: +561-804-4631

Fax: +561-804-4646

E-mail: meister@mathematik.uni-kassel.de

T. Sonar

TU Braunschweig

Carl-Friedrich-Gauß-Fakultät für Mathematik und Informatik

Computational Mathematics

Pockelsstraße 14

D-38106 Braunschweig

Tel.: +531-391-7400

Fax: +531-391-7409

E-mail: t.sonar@tu-bs.de 
ein einfaches Modell auf der Basis von partiellen Differentialgleichungen vor und beschreiben detailliert die numerische Simulation mit Hilfe einer FiniteVolumen-Methode. Dazu wird ein zweidimensionales Modell eines Frühgeborenen trianguliert und das Modell diskretisiert. Zahlreiche numerische Resultate zeigen die Qualität unseres Modells.

Schlüsselwörter Thermoregulation · Finite-Volumen-Verfahren · Wärmeleitungsgleichung · Frühgeborene

Mathematics Subject Classification (2000) 35K05 - 92C50

\section{Einleitung}

Der Begriff Thermoregulation bezeichnet die Fähigkeit eines Organismus, die Körpertemperatur in einem günstigen Bereich aufrechtzuhalten, indem Wärmeabgabe und Wärmeproduktion den äußeren Umständen angepasst werden. Die Thermoregulation des menschlichen Körpers stellt seit Jahrzehnten ein aktives Forschungsgebiet dar. Der Grund dafür sind vielfältige Anwendungen, etwa bei Fragen der Arbeitssicherheit, in denen es um die Auswirkungen äußerer Einflüsse auf die Leistungsfähigkeit und Funktionstüchtigkeit des menschlichen Körpers geht, z.B. im Zusammenhang mit Schutzkleidung für Feuerwehrleute, Frostschutzkleidung oder der Auswirkung längerer Arbeitszeit an Hochöfen, siehe etwa [29] für eine nützliche Übersicht. Weitere interessante Quellen zum Themengebiet Thermoregulation sind insbesondere [25, $28]$.

Modelle menschlicher Thermoregulation beinhalten zwei verschiedene Anteile, ein aktives System sowie ein passives System [16]. Hintergrund dieser Unterscheidung ist, dass das aktive System über das passive System eine kontrollierende Funktion ausübt.

Das aktive System besteht aus den Regulationsmechanismen Kältezittern, Vasomotorik und Schweißbildung [10]. Das Kältezittern ist für die Thermoregulation von Erwachsenen relevant und bezeichnet die Wärmeproduktion in den mit dem Skelett verbundenen Muskeln unter deren aktiver Betätigung. Bei Früh- und Neugeborenen spielt das Kältezittern dagegen eine untergeordnete Rolle, da diese im sogenannten braunen Fettgewebe die Möglichkeit der zitterfreien Wärmebildung besitzen und das Kältezittern weitgehend unterdrückt wird [6]. Die Vasomotorik regelt durch Verengung (Vasokonstriktion) oder Erweiterung (Vasodilatation) der Blutgefäße nahe der Körperoberfläche die Hautdurchblutung, durch die mit Hilfe des Bluttransports der Wärmeaustausch mit der Umgebung gesteuert wird. Das in dieser Arbeit umgesetzte Modell der Vasomotorik stützt sich auf die Untersuchungen in [7]; der Effekt der Vasomotorik wird durch gezielte Veränderung der Hautdurchblutungsrate gesteuert. Die Schweißbildung schließlich regelt insbesondere die Wärmeabgabe. Bei Frühgeborenen spielt Schweißbildung noch keine Rolle $[7,26]$, zumal wir bei Betrachtungen des Inkubators (s.u.) von einem deutlichen Überhitzen der Früh- und Neugeborenen absehen können.

Das passive System besteht aus dem Körper und dessen Eigenschaften, wie z.B. innere Wärmeleitung oder die Größe der Hautoberfläche, sowie 
aus den inneren Wärmebildungs- und übertragungsprozessen, wie etwa die Wärmeproduktion in inneren Organen und die Durchblutung. Auch hier ergeben sich große Unterschiede zwischen Erwachsenen und Früh- bzw. Neugeborenen [7]. So spielt die Wärmeleitung über die Körperoberfläche im letzteren Fall eine größere Rolle, da das Oberflächen-Volumen-Verhältnis etwa um den Faktor drei größer ist als bei Erwachsenen. Desweiteren besitzen frühund neugeborene Kinder im Vergleich zu Erwachsenen eine dünnere Haut sowie eine dünnere isolierende Fettschicht. Die Unterschiede in der Zusammensetzung des Körpers bedingen im Vergleich zu Erwachsenen höhere innere Wärmeleitwerte. Hinzu kommt insbesondere bei Frühgeborenen eine fehlende thermische Reife [5]: Der Körper reagiert auf eine Abkühlung nicht mit einer entsprechenden Steigerungsrate der Wärmeproduktion, so dass Frühgeborene zu Unterkühlung neigen.

Zusammenfassend ist festzuhalten, dass sich sowohl bezüglich des aktiven Systems als auch bezüglich des passiven Systems erhebliche Unterschiede zwischen Erwachsenen und Früh- bzw. Neugeborenen ergeben.

Ein wesentliches Ziel der Therapie Frühgeborener besteht darin, Folgeschäden der frühen Geburt zu minimieren und somit die Voraussetzungen für ein späteres Leben ohne körperliche oder geistige Einschränkungen zu schaffen. Die Möglichkeit einer erfolgreichen Behandlung ist eng mit der Entwicklung und Verbesserung medizintechnischer Geräte verknüpft, wie etwa von Beatmungsgeräten, Monitoringsystemen, Infusions- und Wärmetherapiegeräten.

Von besonderem Interesse im Zusammenhang mit dieser Arbeit sind Inkubatoren, die zu den Wärmetherapiegeräten zählen und die insbesondere Frühgeborenen eine optimale Umgebung für Reifung und Wachstum bieten sollen. Die Wärmetherapie ist, wie bereits angedeutet, für Frühgeborene sehr wichtig, da diese im Vergleich zu Erwachsenen oder reifen Neugeborenen eine wärmere Umgebungstemperatur benötigen, um die Körpertemperatur konstant halten zu können [26]. Zudem können in einem Inkubator weitere Umwelteinflüsse wie Luftfeuchtigkeit, -temperatur und -strömung kontrolliert werden, die für die Thermoregulation bzw. für den Flüssigkeitshaushalt des Frühchens eine Rolle spielen [7]. Da die Empfindlichkeit des Kindes gegenüber Umwelteinflüssen individuellen Unterschieden unterliegt und sich zudem derjenige Bereich des Umgebungsklimas mit zunehmender Reife ändert, in dem das Kind ohne durch Umwelteinflüsse belastet zu werden selbständig die Körpertemperatur aufrechterhalten kann, stellt die optimale Steuerung der Gegebenheiten in einem Inkubator, etwa unter Zuhilfenahme von Daten aus Hauttemperatursensoren, eine komplexe und anspruchsvolle Aufgabe dar. Ein Ziel der numerischen Simulation der Thermoregulation ist, ein Hilfsmittel für die Entwicklung und Verbesserung von Temperaturreglern vor dem Beginn klinischer Tests bereitzustellen.

Jedoch ist nicht nur die Erschaffung einer optimalen Umgebung ein wichtiger Aspekt bei der Therapie Frühgeborener. Ein Hauptgrund für spätere Behinderungen sind Gehirnschädigungen durch Hypoxie, d.h. aufgrund von Sauerstoffunterversorgung des Gehirngewebes [18]. Eine Hypoxie kann bei Neu- und vor allem bei Frühgeborenen vor oder während der Geburt auftreten, meist in Folge eines asphyktischen Anfalls, d.h. bei Atemstillstand oder einer 
Einschränkung der Atmung und Sauerstoffversorgung. Speziell bei sogenannten Steißlagengeburten kann es im Zuge der Geburtsphase zu einem Abklemmen der Nabelschnur im Geburtskanal aufgrund der simultanen Präsenz von Nabelschnur und Kopf kommen. Die hierdurch hervorgerufene drastische Reduktion des Sauerstoffzuflusses stellt eine weitere mögliche Ursache einer Hypoxie dar.

Experimentelle Studien haben gezeigt, dass derartige Schädigungen des Gehirngewebes nicht spontan auftreten, sondern sich über einen Zeitraum von mehreren Tagen entwickeln [13]. Der Umfang der Schädigungen des Gewebes ist dabei im wesentlichen von der Gewebetemperatur abhängig [8]; eine Absenkung der Gewebetemperatur um 2-3 K ${ }^{1}$ verhindert schwerwiegende Schädigungen. Es ergibt sich die Frage, ob es möglich ist, etwa mittels eines Kühlungshelms gezielt die Temperatur des Gehirngewebes zu senken, um auf diese Weise Hirnschädigungen zu verhindern, ohne dabei andere Körperkompartimente zu unterkühlen.

An dieser Stelle sei noch einmal auf die Unterschiede zwischen der Thermoregulation von Erwachsenen einerseits und früh- bzw. neugeborenen Kindern andererseits hingewiesen. Bei Erwachsenen ist die Idee der Nutzung eines derartigen Kühlungshelmes redundant: Der Körper würde in einem der Kühlung entsprechenden Maße Wärme produzieren. Im Extremfall würden bei intensiver Kühlung Körperfunktionen, die nicht überlebensnotwendig sind, abgeschaltet werden, um die Gehirntemperatur zu erhalten. Im Zusammenhang mit Früh- und Neugeborenen sind die körperlichen Voraussetzungen im Hinblick auf die besagte Fragestellung völlig anders. Zum einen ergibt sich durch die andere Körpergeometrie sowie durch eine andere Körperzusammensetzung eine erhöhte Empfindlichkeit gegenüber äußeren Kühlungseinfüssen. Zum anderen fehlt insbesondere den Frühgeborenen die bei den Erwachsenen voll ausgebildete thermische Reife. Aufgrund der Kombination dieser Eigenschaften ist die besagte Fragestellung sinnvoll.

Da es aufgrund technischer Beschränkungen und ethischer Aspekte nicht möglich ist, die Temperatur im Gehirn mit hinreichender Genauigkeit zu messen [7], gehen wir dieser Frage mit Hilfe eines zu entwickelnden mathematischen Modells nach. In diesem Zusammenhang sei ebenfalls angemerkt, dass aus medizinischer Sicht die Wechselwirkung der an der Thermoregulation beteiligten Prozesse nicht so gut verstanden ist, dass man auf Erfahrungswerte zurückgreifen kann. Diese Tatsache spricht ebenfalls für den Einsatz eines mathematischen Modells, in dem die bekannten Teilaspekte vereint sind.

Das Modell besteht letztlich aus einer sich ergebenden speziellen BioWärmeleitungsgleichung, die die Wärmeverteilung im idealisierten Körper

\footnotetext{
${ }^{1}$ Nach dem internationalen Einheitensystem, auch SI für Système International d'Unités genannt, ist es üblich, Temperaturdifferenzen in $K$ (für Kelvin) anzugeben. Temperaturdifferenzen können vom Prinzip her auch in Grad Celsius angegeben werden, das den gleichen Skalenabstand aufweist wie die Kelvin-Skala. Der Nullpunkt der Celsius-Skala bezieht sich auf den Gefrierpunkt von Wasser beim Normaldruck, d.h. auf den mittleren Luftdruck auf Meereshöhe, während der Nullpunkt der Kelvin-Skala beim absoluten Nullpunkt liegt, d.h. bei $-273,16^{\circ} \mathrm{C}$. In Deutschland und den meisten anderen Staaten ist die Benutzung des SI im amtlichen oder geschäftlichen Schriftverkehr gesetzlich vorgeschrieben.
} 
eines Früh- oder Neugeborenen beschreibt. Zur Berechung der Wärmeverteilung wird der idealisierte Körper hinreichend fein in Rechenzellen aufgeteilt. Je nach deren Lage im Körper ergeben sich auf diesen Rechenzellen entsprechend der Wärmeproduktion in inneren Organen sowie entsprechend der Durchblutung Wärmequellen. Zusätzlich liegen durch die Wärmeleitungseigenschaften der Körpergewebe Wärmeflüsse zwischen den Rechenzellen vor. Die Auswertung dieser Einflüsse, die alle durch die modellierte Wärmeleitungsgleichung beschrieben werden, geschieht mit Hilfe eines Finite-Volumen-Verfahrens, das im entprechenden Abschnitt vorgestellt wird, wobei die "finiten Volumen" den Rechenzellen entsprechen. Da insbesondere Situationen von Interesse sind, in denen der Zustand des Frühchens bzw. des Neugeborenen im thermischen Sinne stabil ist, betrachten wir zeitlich stationäre Zustände der Wärmeverteilung.

Der Inhalt dieser Arbeit gliedert sich wie folgt. Die detaillierte Beschreibung der Modellierung der Thermoregulation von Früh- und Neugeborenen befindet sich im ersten Abschnitt. Das numerische Verfahren wird im darauffolgenden Abschnitt beschrieben, gefolgt von der Darstellung verschiedener Simulationsergebnisse sowie deren Diskussion. Zum Abschluss der Arbeit erfolgen eine Zusammenfassung sowie Danksagungen.

\section{Modellbildung}

Zu Beginn der Modellbildung ist darzulegen, was von dem Modell erwartet wird.

- Das Modell soll bei selektiver Kühlung des Kopfes eine Aussage über die Temperaturverteilung im gesamten Körper sowie insbesondere eine detaillierte Darstellung der Temperaturverteilung im Kopf liefern.

- Das Modell soll die bekannten thermoregulatorischen Mechanismen enthalten.

- Das Modell muss, da im klinischen Bereich Frühgeborene unterschiedlicher Schwangerschaftsdauern behandelt werden sollen, die thermische Reife berücksichtigen.

In der Fachliteratur, siehe [25,28,29], gibt es im wesentlichen drei Modelltypen, die im Zusammenhang mit mathematischen Beschreibungen der Thermoregulation Verwendung finden:

1. Kern-Schale-Modelle,

2. Segment- und Zylindermodelle,

3. Mehrdimensionale Modelle.

Kern-Schale-Modelle enthalten als wesentliche Komponenten eine Kerntemperatur, während der Wärmeaustausch mit der Umgebung sowie physiologische Aspekte mit Hilfe einer Schale rund um den Kern modelliert werden. Der Körper wird dabei durch eine simple Geometrie wie z.B. einen Zylinder dargestellt, der in Kern und Schale aufgeteilt ist. Diese Modelle ergeben lediglich Durchschnittstemperaturen für Kern und Schale des ganzen Körpers. 
Segment- und Zylindermodelle modellieren typischerweise verschiedene Körpersegmente, d.h. Kopf, Rumpf und Gliedmaßen, durch unverbundene einfache geometrische Objekte, typischerweise durch Zylinder. Daraus ergibt sich zunächst die Konsequenz, dass Wärmeleitung über das Gewebe zwischen den Segmenten nicht stattfindet; der Wärmeaustausch zwischen den Körpersegmenten geschieht bei diesen Modellen ausschließlich über den Blutfluss. Diese Herangehensweise ist auf der Modellierungsebene durchaus adäquat, da der Wärmeaustausch durch den Blutfluss dominant gegenüber der Wärmeleitung durch das Gewebe ist. Segment- und Zylindermodelle sind ähnlich wie Kern-Schale-Modelle jedoch lediglich darauf ausgelegt, Durchschnittstemperaturen für Kern und Schale jedes Körpersegmentes anzugeben.

Eine Weiterentwicklung der Segment- und Zylindermodelle ist das Modell von Bußmann [7]. Dieses Modell beinhaltet - wie bei Segment- und Zylindermodellen - neben Modellen für wesentliche Thermoregulatoren und Blutfluss Segmente für Kopf, Rumpf und Glieder, für die im Bußmann-Modell zusätzlich die Möglichkeit von radialen Temperaturvariationen innerhalb der Körpersegmente gegeben ist. Dabei werden radiale Durchschnittswerte benutzt, d.h. allen Punkten im gleichen radialen Abstand von den Achsen der Segmente wird die gleiche Temperatur zugeordnet. Für unsere Bedürfnisse ist dieses Modell jedoch ebenfalls nicht ausreichend, da es lokal unterschiedliche thermische Randbedingungen, die sich durch das Anlegen eines Kühlungshelms ergeben, sowie lokale Gewebeeigenschaften, wie etwa im Kopfbereich durch den lokal sehr unterschiedlichen Knochenanteil, nicht berücksichtigt.

Eine adäquate Berücksichtigung lokal verschiedener thermischer Randbedingungen und Gewebeeigenschaften sowie die insbesondere im Hals- und Kopfbereich möglicherweise doch ausschlaggebende Wirkung der Wärmeleitung des Gewebes lässt sich nur in einem mehrdimensionalen Modell realisieren. Das erste mehrdimensionale Modell der Thermoregulation von Frühund Neugeborenen, das den genannten Anforderungen entspricht, wurde in $[11,12,17]$ entwickelt und in [1-4] untersucht und erweitert. In diesem Abschnitt stellen wir bis auf die Realisierung durch das numerische Verfahren die einzelnen Modellkomponenten vor.

\subsection{Die Bio-Wärmeleitungsgleichung}

In diesem Abschnitt diskutieren wir die sogenannte Bio-Wärmeleitungsgleichung, die der Berechnung der Temperaturverteilung im Körper des Früh- bzw. Neugeborenen zugrundeliegt:

$$
c(x) \rho(x) \frac{\partial T}{\partial t}(x, t)=\nabla_{x} \cdot\left(\lambda(x) \nabla_{x} T\right)(x, t)+f(x, t) .
$$

Dabei gilt unter Verwendung der gängigen SI-Einheiten:

$-T(x, t)$ ist die zu berechnende Temperatur am Ort $x \in \mathcal{D}$ zum Zeitpunkt $t>0,[T]=K$,

$-\lambda(x)$ ist die Wärmeleitfähigkeit des Gewebes, $[\lambda]=W /(m K)$,

$-\rho(x)$ ist die Dichte des Gewebes, $[\rho]=k g / \mathrm{m}^{3}$,

$-c(x)$ ist dessen spezifische Wärmekapazität, $[c]=J /(k g K)$, 
- $f(x, t)$ bezeichnet lokale Wärmeproduktion und Wärmeverteiler, $[f]=$ $J /\left(m^{3} s\right)$.

Die Gleichung (1) wurde als Gleichung für die Entwicklung der Temperaturverteilung in einem von feinen Kapillaren durchzogenen durchbluteten Gewebe von Pennes [21] aufgestellt. Physikalisch gesehen beschreibt (1) die Wärmeleitung in einem ruhenden, isotropen Festkörper, d.h. in einem Körper, bei dem sich Wärme nach allen Richtungen gleich gut ausbreiten kann. Der Term

$$
\nabla_{x} \cdot\left(\lambda \nabla_{x} T\right)(x, t)
$$

beinhaltet dabei die Wärmeflüsse aufgrund der Wärmeleitung des Gewebes.

Wie bereits angedeutet interessieren uns thermisch stabile Zustände, d.h. in diesem Falle gilt in (1)

$$
\frac{\partial T}{\partial t}(x, t)=0 .
$$

Daher spielen im thermisch stabilen Zustand die Werte von $c$ und $\rho$ keine Rolle, da sie endlich sind und mit Null multipliziert werden. Im Abschnitt über die Konstruktion des numerischen Verfahrens werden wir sehen, wie die angestrebte Beziehung (2) algorithmisch ausgenutzt wird.

Im Hinblick auf eine vollständige Beschreibung der mathematischen Aufgabenstellung muss die Gleichung (1) noch durch Angabe von Randbedingungen sowie von Anfangsbedingungen ergänzt werden: Bezeichnet $\mathcal{D}$ das vorgegebene Rechengebiet, so werden Randbedingungen auf dem Rand von $\mathcal{D}$, d.h. auf $\partial \mathcal{D}$, vorgegeben, während Anfangsbedingungen dem Zustand entsprechen, der zum Anfangszeitpunkt $t=0$ einer zeitlichen Evolution auf dem gesamten Rechengebiet vorzugeben ist. Da innerhalb eines Inkubators die Lufttemperatur sowie die Temperatur der Rückenunterlage des Kindes kontrolliert werden können, setzen wir Dirichlet-Randbedingungen an und geben somit auf $\partial \mathcal{D}$ die Temperatur vor. Die gleiche Art der Randbedingung ist bei der Benutzung eines Kühlungshelms verfügbar. Da sich die stationäre Temperaturverteilung letztlich durch die Randwerte ergibt, spielen die Anfangswerte bei der Berechung stationärer Zustände hier keine wichtige Rolle: Mit Hilfe von (2) werden wir die Gleichung (1) später für die Konstruktion unseres numerischen Verfahrens weiter vereinfachen, so dass Zustände, die sich nach Vorgabe von Anfangsdaten auf dem Wege der Berechnung zu einem stationären Zustand hin ergeben, keine physikalische Bedeutung besitzen. Im Gegensatz hierzu repräsentiert das numerische Resultat im konvergenten Endzustand eine Approximation der durch das Modell beschriebenen Realität.

Zur konkreten Anwendung der Gleichung (1) auf die Temperaturverteilung eines früh- oder neugeborenen Kindes müssen abschließend noch die Körpergeometrie sowie der innere Aufbau des Körpers festgelegt werden. Zudem muss die Größe $\lambda$ angegeben sowie die Funktion $f$ determiniert werden.

\subsection{Modellierung des Körpers}

Wir verwenden ein räumlich zweidimensionales idealisiertes Modell des Körpers eines früh- bzw. neugeborenen Kindes. Der Gesamtkörper ist in drei 


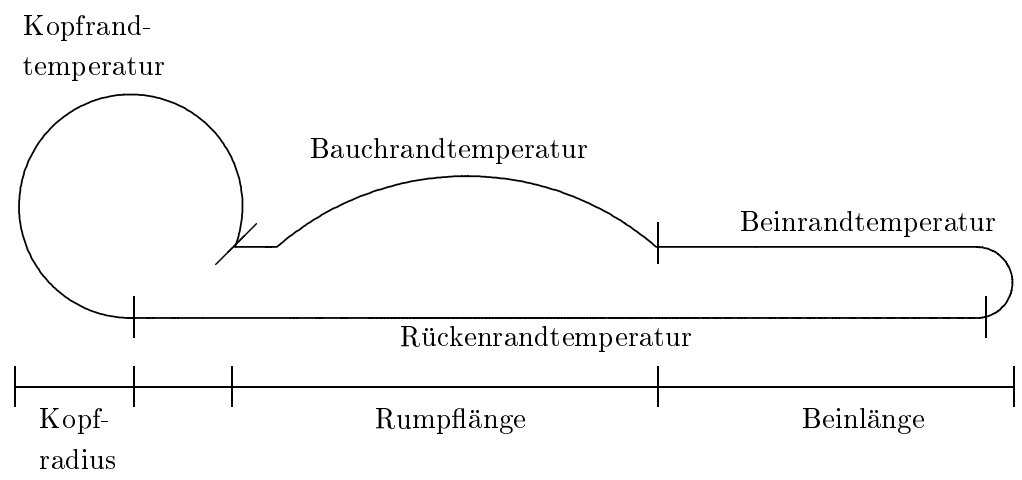

Abb. 1 Aufbau des zweidimensionalen idealisierten Körpers des Früh- bzw. Neugeborenen zusammen mit wählbaren Randtemperaturbereichen

Tabelle 1 Statistisch ermittelte Größenverhältnisse in Abhängigkeit vom Gewicht des Kindes in $[g]$

\begin{tabular}{ll}
\hline Abmessung $[m]$ & Formel \\
\hline Körperlänge & $0.178+0.255 g-0.107 g^{2}+0.027 g^{3}-0.003 g^{4}$ \\
Kopfumfang & $0.045+0.596 *$ Körperlänge \\
Beinlänge & $0.396 *$ Körperlänge -0.01 \\
Beindicke & $0.1 *$ Körperlänge -0.009 \\
Bauchhöhe & Beindicke \\
Rumpflänge & $0.64 *$ Körperlänge $-2.0 *$ Kopfradius +0.009 \\
Halslänge & $0.1 *$ Rumpflänge \\
\hline
\end{tabular}

Bereiche unterteilt: Kopf, Rumpf und Beine. Die idealisierte Geometrie des Körpers sowie die Bereiche, in denen entsprechende Randtemperaturen angelegt werden können, ist in Abbildung 1 dargestellt.

Die Geometrie des Körpers, d.h. alle Koordinaten und Abmessungen, werden durch das Gewicht des Kindes berechnet. Die thermische Reife kann statistisch mit dem Körpergewicht in Verbindung gebracht werden; dieses wiederum korelliert statistisch mit der Körpergröße und dessen Geometrie, so dass die thermische Reife über die Darstellung des Körpers ins Modell einbezogen wird. Die implementierten Größenverhältnisse sind in Tabelle 1 dargestellt.

Jeder Bereich des Körpers ist wiederum in Schichten unterteilt, siehe Abbildung 2 für den Fall des Kopfes. Im Einzelnen gilt:

- Kopf: Haut (1mm), Fett (1mm), Knochen/Schädel (2mm), Kern.

- Rumpf, Beine: Haut (1mm), Fett (1mm), Kern.

Die Wärmeleitfähigkeit $\lambda(x)$ wird entsprechend des am Ort $x$ vorhandenen Gewebetyps gesetzt [9], siehe Tabelle 2 .

Grundsätzlich ist es möglich, eine genauere oder dreidimensionale Darstellung des Körpers zu benutzen. Die gewählte Herangehensweise ist zum einen als Zwischenschritt in Richtung eines genaueren, dreidimensionalen Modells 
Tabelle 2 Ortsabhängige Parameterwerte für $\lambda(x)$

\begin{tabular}{ll}
\hline Gewebetyp & $\lambda$ \\
\hline Haut & 0.35 \\
Fett & 0.21 \\
Knochen & 0.4 \\
Kern & 0.51 \\
\hline
\end{tabular}

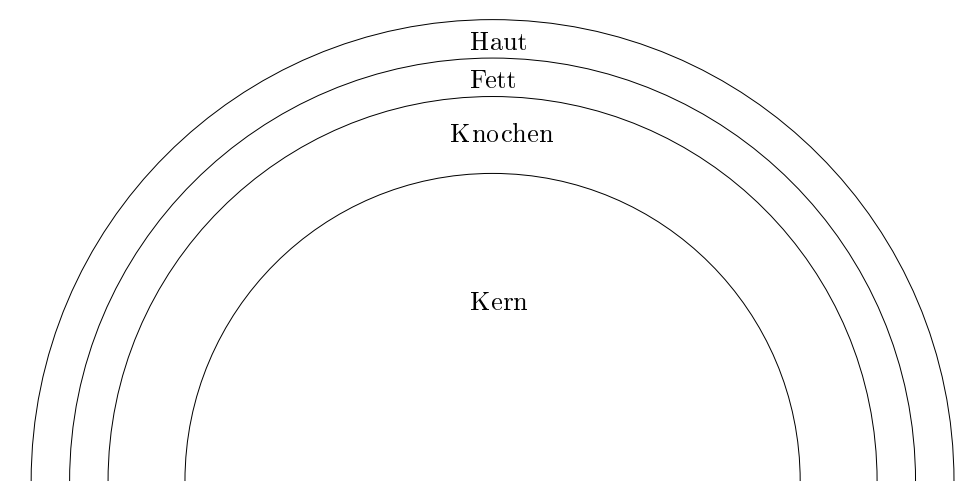

Abb. 2 Gewebeschichten im Kopf

des Körpers zu sehen. Auf der anderen Seite liefert das präsentierte Modell bereits klare Hinweise im Sinne der definierten Ziele und stellt daher im Sinne der Aufgabenstellung einen angemessenen Weg dar. In diesem Zusammenhang sei darauf hingewiesen, dass zum gegenwärtigen Zeitpunkt kein zugänglicher Datensatz existiert, der die Geometrie des Körpers eines frühoder neugeborenen Kindes genau wiedergibt und für eine numerische Simulation nutzbar wäre, d.h. man ist zur Zeit auf idealisierte Ansätze wie das hier präsentierte Modell angewiesen. Ohne eine höhere Detailtreue ist jedoch nicht zu erwarten, dass etwa lediglich die Berücksichtigung einer weiteren Raumdimension qualitativ andere Ergebnisse erbringt.

\subsection{Modellierung des Quellterms}

Es steht nun noch die Modellierung des Quellterms $f$ aus, der lokale metabolische Wärmeproduktion und Wärmeverteilung beschreibt. Besagte Einflüsse können additiv aufgeteilt werden, letztlich gilt

$$
f(x, t)=Q_{M}(x)+Q_{B}(x, t) .
$$

Im weiteren definieren wir die lokalen Quellterme $Q_{M}$ und $Q_{B}$. 
Tabelle 3 Metabolische Wärmeproduktion in $\left[\frac{W}{m^{2}}\right]$

\begin{tabular}{llll}
\hline & Kopf & Rumpf & Bein \\
\hline Haut & 0.0 & 0.0 & 0.0 \\
Fett & 0.0 & 0.0 & 0.0 \\
Knochen & 0.0 & 0.0 & 0.0 \\
Kern & 4500 & 1800 & 600 \\
\hline
\end{tabular}

Tabelle $4 K(x)$-Faktoren

\begin{tabular}{llll}
\hline & Kopf & Rumpf & Bein \\
\hline Haut & 1.0 & 1.0 & 0.9 \\
Fett & 1.0 & 1.0 & 0.9 \\
Knochen & 1.0 & 1.0 & 0.9 \\
Kern & 1.0 & 1.0 & 0.9 \\
\hline
\end{tabular}

\subsubsection{Metabolische Wärmeproduktion}

Die Wärmebildung entspricht der Wärmeproduktion im braunen Fettgewebe sowie in inneren Organen, insbesondere im Gehirn. Verschiedene Studien belegen, dass in Früh- und Neugeborenen zwischen 30 und 60 Prozent der im gesamten Körper produzierten Wärme im Gehirn gebildet wird, siehe [7,15].

Wir bezeichnen aufgrund des dargestellten Hintergrundes die Wärmebildung als metabolische Wärmeproduktion und bezeichnen den entsprechenden Quellterm als $Q_{M}$. Die Wärmeproduktion $Q_{M}$ wird in den einzelnen Bereichen bzw. Gewebeschichten als konstant und homogen verteilt angesehen. Sie tritt nur im Kern des Kindes auf, ist im Kopfkern - entsprechend der thermischen Bedeutung des Gehirns - am stärksten und fällt zu den Beinen ab, siehe Tabelle 3; vergleiche auch $[7,17]$.

\subsubsection{Blutfluss}

Der Blutfluss ist neben der Wärmeleitung das wichtigste Mittel der Wärmeverteilung. Abhängig von den Bereichen - modelliert durch Faktoren $K(x)-$ sowie von den durchschnittlichen Durchblutungsraten der modellierten Gewebetypen - dargestellt durch Blutflussraten $B F(x)$ - setzen wir den Quellterm $Q_{B}$ an als

$$
Q_{B}(x, t)=\rho_{B L} \cdot c_{B L} \cdot K(x) \cdot B F(x) \cdot\left(T_{B L}(t)-T(x, t)\right) .
$$

Die Größen

$$
\rho_{B L}=1060 \frac{\mathrm{kg}}{\mathrm{m}^{3}} \quad \text { und } \quad c_{B L}=3840 \frac{\mathrm{J}}{\mathrm{kgK}}
$$

bezeichnen dabei die Blutdichte bzw. die spezifische Wärmekapazität des Blutes, die Wahl der Größen $K(x)$ und $B F(x)$ entnehme man den Tabellen 4 und 5; vergleiche $[7,17,30]$.

Der Ansatz (4) bedarf der weiteren Erläuterung. Wie bereits erwähnt, liegt dem klassischen Weg der Modellierung eines in feinen Kapillaren mit 
Tabelle 5 Blutfluss $B F(x)$ in $\left[\frac{1}{s}\right]$

\begin{tabular}{llll}
\hline & Kopf & Rumpf & Bein \\
\hline Haut & $1.25 \cdot 10^{-3}$ & $1.25 \cdot 10^{-3}$ & $1.25 \cdot 10^{-3}$ \\
Fett & 0.0 & 0.0 & 0.0 \\
Knochen & 0.0 & 0.0 & 0.0 \\
Kern & $3.3 \cdot 10^{-3}$ & $7 \cdot 10^{-3}$ & $1.25 \cdot 10^{-3}$ \\
\hline
\end{tabular}

Blut durchflossenen Gewebes die Arbeit von Pennes [21] zugrunde. Ausgehend von Pennes' Betrachtungen ergeben sich Blutflussmodelle, die abgesehen von Feinheiten die folgende Betrachtung zugrundelegen, vergleiche etwa $[4,29]$.

Die Idee ist, dass der Körper über die Arterien von einem zentralen Blutreservoir aus versorgt wird. Das arterielle Blut fließt in die von den Arterien versorgten Körperteile und tauscht dort Wärme mit dem Gewebe aus: Es dient dort als Wärmequelle und nimmt gleichzeitig, d.h. in einem Ausgleichsprozess, die Temperatur des umliegenden Gewebes an. Anschließend fliesst es durch die Venen in das zentrale Blutreservoir zurück. Dort wird es vermischt und es findet ein Temperaturausgleich statt, d.h. das Blut im zentralen Blutreservoir erhält eine homogene Temperatur bevor es durch die Arterien wieder weitertransportiert wird.

Entsprechend berechnen wir die Temperatur des zentralen Blutreservoirs $T_{B L}$ abhängig vom betrachteten Körperteil sowie von den Durchblutungsraten der Gewebe als

$$
T_{B L}(t)=\frac{\int_{\mathcal{D}} K(x) B F(x) T(x, t) d x}{\int_{\mathcal{D}} K(x) B F(x) d x} .
$$

Grob gesagt handelt es sich bei $T_{B L}$ aus (5) also um den gewichteten Mittelwert der Temperatur des venösen Blutes. An dieser Stelle wird deutlich, dass wir bei unserer Modellierung bereits auf stationäre Zustände abzielen, da bei der Bezugnahme auf venöses Blut ein Vorgriff auf eine an sich noch zu berechnende Temperaturverteilung stattfindet; der Term (5) ist nur dann im Sinne des Modells korrekt, wenn die Temperaturverteilung zeitlich invariant ist.

\subsubsection{Vasomotorik}

Wie die Untersuchungen in [7] gezeigt haben, ist der Effekt der Vasomotorik in einem thermisch neutralen Bereich bei Frühgeborenen vernachlässigbar. Anders ist die Situation bei relativ extremer lokaler Hitze- oder Kältebelastung.

Nach den Untersuchungen in [?] ergibt sich bei großer Hitzebelastung eine maximale Vasodilatation, während sich bei starker Kältebelastung eine maximale Vasokonstriktion ergibt; in dem Zwischenbereich kann eine lineare Abhängigkeit des Effekts der Vasomotorik unterstellt werden. Der Effekt der maximalen Vasokonstriktion wird nach der in [7] angefertigten quantitaven Analyse in dem numerischen Testfall, der dem Anlegen eines Kühlungshelmes entspricht, durch Modifikation der Durchblutungsrate der am Kopf befindlichen Hautschicht mittels des Faktors 1/100 berücksichtigt. 


\section{Das Finite-Volumen-Verfahren}

Zur Vorbereitung der Anwendung unseres Finite-Volumen-Verfahrens integrieren wir die Bio-Wärmeleitungsgleichung (1) über ein beliebig, aber fest ausgewähltes, endliches und nicht leeres Kontrollvolumen $\sigma \subset \mathcal{D}$, auch Zelle oder Box genannt:

$$
\int_{\sigma} c(x) \rho(x) \frac{\partial T}{\partial t}(x, t) d x=\int_{\sigma} \nabla_{x} \cdot\left(\lambda(x) \nabla_{x} T\right)(x, t) d x+\int_{\sigma} f(x, t) d x .
$$

Da uns, wie bereits besprochen, in dieser Arbeit lediglich die durch (2) definierten stationären Zustände interessieren, setzen wir o.B.d.A. $c(x) \rho(x) \equiv$ 1 .

Es bezeichne nun $|\sigma|$ den Flächeninhalt des Kontrollvolumens $\sigma$. Nach Division durch $|\sigma|$ und Anwendung des Satzes von Gauß auf den ersten Term der rechten Seite von (6) erhalten wir die Grundlage unseres Finite-VolumenVerfahrens, nämlich eine Evolutionsgleichung für Zellmittelwerte:

$$
\begin{aligned}
& \frac{d}{d t}\left[\frac{1}{|\sigma|} \int_{\sigma} T(x, t) d x\right] \\
& =\frac{1}{|\sigma|} \int_{\partial \sigma} \lambda(x) \nabla_{x} T(x, t) \cdot \mathbf{n}(x) d s+\frac{1}{|\sigma|} \int_{\sigma} f(x, t) d x .
\end{aligned}
$$

Dabei ist $\mathbf{n}(x)$ der äußere Einheitsnormalenvektor im Punkt $x \in \partial \sigma$.

Das Finite-Volumen-Verfahren besteht aus einer Diskretisierungsvorschrift für die Evolutionsgleichung (7), die auf allen betrachteten Kontrollvolumina $\sigma$ des Rechengebietes gelöst werden muss.

Im angestrebten stationären Zustand ergibt sich nach (2)

$$
\frac{d}{d t}\left[\frac{1}{|\sigma|} \int_{\sigma} T(x, t) d x\right]=0 .
$$

Benutzen wir nicht wie in der Herleitung von (7) die Vereinfachung $c(x) \rho(x) \equiv$ 1, so sind nach Gleichung (6) andere Zellmittelwerte, nämlich

$$
\frac{1}{|\sigma|} \int_{\sigma} c(x) \rho(x) T(x, t) d x
$$

zu wählen. Da in diesem Fall durch das Verfahren die zeitliche Evolution der Variable $\tilde{T}(x, t):=c(x) \rho(x) T(x, t)$ beschrieben wird, ist konsequenterweise die rechte Seite der Ausgangsgleichung in Abhängigkeit von $\tilde{T}$ zu formulieren, wodurch sich ein zusätzlicher Quellterm ergibt. Wie in $[2,3]$ verdeutlicht wird, ist diese Vorgehensweise für die Approximation instationärer Zustände notwendig.

Aus physikalischer Sicht spiegelt die Bilanzgleichung (7) den ersten Hauptsatz der Thermodynamik wider, der besagt, dass die zeitliche Änderung der auf ein Gebiet $\sigma$ bezogenen inneren Energie, hier

$$
\frac{d}{d t} \int_{\sigma} T(x, t) d x
$$


im Gleichgewicht mit der über den Rand zugeführten Wärmeenergie, dargestellt durch

$$
\int_{\partial \sigma} \lambda(x) \nabla_{x} T(x, t) \cdot \mathbf{n}(x) d s,
$$

und der in $\sigma$ geleisteten Arbeit

$$
\int_{\sigma} f(x, t) d x
$$

steht. Die spezielle Form der in (8) vorliegenden Wärmeflüsse repräsentiert hierbei das Fouriersche Grundgesetz des molekularen Wärmetransports, das den Zusammenhang zwischen dem Wärmefluß und den Temperaturgradienten beschreibt. Der erste Term auf der rechten Seite der Bilanzgleichung (7) verkörpert offensichtlich einen reinen Wärmetransport, da die Darstellung in Form eines Randintegrals stets den Transport von Wärmeenergie zwischen benachbarten Kontrollvolumina über deren gemeinsame Ränder beschreibt. Dagegen stellt der zweite Term lokale Produktions- respektive Dissipationseffekte dar, die in der partiellen Differentialgleichung (1) nicht in Divergenzform vorliegen und folglich in (7) nicht als Randintegral dargestellt werden können. Eine derart lokale Wirkung ist bei der metabolischen Wärmeproduktion zu erwarten. Dem Blutfluß würde man jedoch den Effekt einer reinen Wärmeverteilung zuordnen, der, integriert über dem Gesamtkörper stets identisch verschwindet. Das heißt: Blutfluß verteilt Wärme und erzeugt sie nicht. Es ist an dieser Stelle daher zu bemerken, dass die vorgenommene Modellierung diesem Effekt gerecht wird, denn es gilt

$$
\begin{aligned}
& \int_{\mathcal{D}} Q_{B}(x, t) d x \\
& \stackrel{(4)}{=} \rho_{B L} \cdot c_{B L} \cdot \int_{\mathcal{D}} K(x) B F(x)\left(T_{B L}(t)-T(x, t)\right) d x \\
& =\rho_{B L} \cdot c_{B L} \cdot \underbrace{T_{B L}(t) \int_{\mathcal{D}} K(x) B F(x) d x}_{\stackrel{(5)}{=} \int_{\mathcal{D}} K(x) B F(x) T(x, t) d x}-\int_{\mathcal{D}} K(x) B F(x) T(x, t) d x] \\
& =0 .
\end{aligned}
$$

Das verwendete Blutflußmodell kommt daher seiner wärmeverteilenden Funktion nach, obwohl keine Divergenzform des zugehörigen Terms $Q_{B}$ vorliegt.

Der erste Schritt zur Herleitung der angestrebten Finite-Volumen-Methode liegt in der Zerlegung des Gebietes $\mathcal{D}$ in endlich viele disjunkte und abgeschlossene Zellen $\sigma_{i} \subset \overline{\mathcal{D}}:=\mathcal{D} \cup \partial \mathcal{D}, i=1, \ldots, n$, derart, dass

$$
\bigcup_{i=1}^{n} \sigma_{i}=\overline{\mathcal{D}}
$$

erfüllt ist, sowie für $\stackrel{\circ}{\sigma}_{i}:=\sigma_{i} \backslash \partial \sigma_{i}$ die Bedingung

$$
{\stackrel{\circ}{\sigma_{i}}}_{i} \cap{\stackrel{\circ}{\sigma_{j}}}=\emptyset, \quad i, j \in\{1, \ldots, n\}, \quad i \neq j,
$$


gilt. Der zweite Schritt liegt anschließend in der Approximation der Evolutionsgleichung für jedes vorliegende Kontrollvolumen. Um eine hohe Flexibilität bei der Raumdiskretisierung zu erlangen, nutzen wir eine konforme Triangulierung. Diese Gitter bestehen aus einer Menge abgeschlossener Dreiecke $D_{i} \subset \overline{\mathcal{D}}, i=1, \ldots, N$, die paarweise disjunkt im Sinne von

$$
\stackrel{\circ}{D}_{i} \cap \stackrel{\circ}{D}_{j}=\emptyset, \quad i, j \in\{1, \ldots, N\}, \quad i \neq j,
$$

sind, der Bedingung

$$
\bigcup_{i=1}^{N} D_{i}=\overline{\mathcal{D}}
$$

genügen und keine sogenannten hängenden Knoten aufweisen, d.h. jede Randkante eines Dreiecks ist entweder Randkante genau eines benachbarten Dreiecks oder Teil des Gebietsrandes $\partial \mathcal{D}$. Das Auftreten der in Bild 4 (rechts) dargestellten Situation ist somit untersagt. Eine unter diesen Bedingungen erzeugte Triangulierung des Körpers $\overline{\mathcal{D}}$ ist in Bild 3 dargestellt, während Bild 4 (links) die Diskretisierung des Kopfbereiches widerspiegelt. Aufgrund der unterschiedlichen Körperkompartimente sowie der zu erwartenden starken Temperaturvariationen in den Körperrandschichten wurde senkrecht zum Rand $\partial \mathcal{D}$ eine feinere Auflösung im Vergleich zum Kernbereich gewählt. Die somit vorliegenden Dreiecke können direkt als Zellen für das Finite-VolumenVerfahren genutzt werden. Derartige Ansätze werden als Primärnetzmethoden bezeichnet.

Um bei der Auswertung des auf die Zelle $\sigma_{i}$ bezogenen Randintegrals

$$
\int_{\partial \sigma_{i}} \lambda(x) \nabla_{x} T(x, t) \cdot \mathbf{n}(x) d s
$$

möglichst viele Raumrichtungen zu berücksichtigen, und zudem stets eine eindeutige und intuitiv verständliche Berechnungsvorschrift für die auftretenden Temperaturgradienten zu erzielen, nutzen wir eine sogenannte Sekundärnetzmethode. In unserem Fall wird hierzu ein duales Gitter durch Verbindung des Schwerpunktes mit den Seitenhalbierenden jedes Dreieckes erzeugt. Eine exemplarische Darstellung der hieraus resultierenden Zellen $\sigma_{i}$ findet man in Bild 5. In der Regel weisen benachbarte Boxen $\sigma_{i}$ und $\sigma_{j}$ zwei gemeinsame Randgeradenstücke auf, die wir mit $\ell_{i j}^{k}, k=1,2$ bezeichnen, siehe Bild 6 . Im Fall einer Randzelle $\sigma_{i}$, d.h. $\partial \sigma_{i} \cap \partial \mathcal{D} \neq \emptyset$, existieren zwei Nachbarzellen, zu denen $\sigma_{i}$ nur eine gemeinsame Randkante besitzt. Um eine weitgehend einheitliche Darstellung zu gewährleisten, weisen wir der fehlenden Randkante die Länge Null zu, d.h. $\left|\ell_{i j}^{2}\right|=0$. Wie in der Abbildung 7 verdeutlicht, werden die bei einer Randzelle $\sigma_{i}$ vorliegenden zwei Geradenstücke $\partial \sigma_{i} \cap \partial \mathcal{D}$ mit $\ell_{\sigma_{i}}^{k}$, $k=1,2$, bezeichnet.

Bezeichnen wir mit $\mathcal{N}(i)$ die Menge aller Indizes $j$ der zu $\sigma_{i}$ gehörenden Nachbarboxen $\sigma_{j}$, so läßt sich die integrale Form der Evolutionsgleichung (7) unter Verwendung der Zellmittelwerte

$$
T_{i}(t):=\frac{1}{\left|\sigma_{i}\right|} \int_{\sigma_{i}} T(x, t) d x
$$




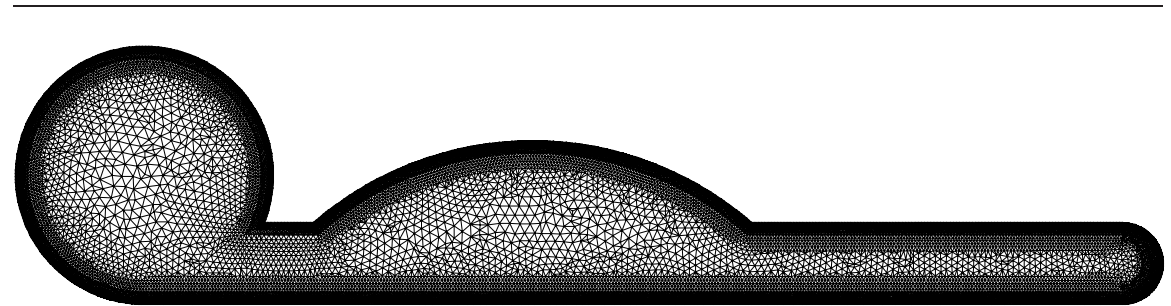

Abb. 3 Triangulierung des zweidimensionalen idealisierten Körpers des Frühbzw. Neugeborenen

in der Form

$$
\begin{aligned}
\frac{d}{d t} T_{i}(t)= & \frac{1}{\left|\sigma_{i}\right|} \sum_{j \in \mathcal{N}(i)} \sum_{k=1}^{2} \int_{\ell_{i j}^{k}} \lambda(x) \nabla_{x} T(x, t) \cdot \mathbf{n}_{i j}^{k} d s \\
& +\frac{1}{\left|\sigma_{i}\right|} \sum_{k=1}^{2} \int_{\ell_{\sigma_{i}}^{k}} \lambda(x) \nabla_{x} T(x, t) \cdot \mathbf{n}_{\sigma_{i}}^{k} d s \\
& +\frac{1}{\left|\sigma_{i}\right|} \int_{\sigma_{i}} f(x, t) d x
\end{aligned}
$$

für $i=1, \ldots, n$ darstellen. Analog zu den inneren Kanten $\ell_{i j}^{k}$ weisen wir den Randkanten $\ell_{\sigma_{i}}^{k}$ jeweils die Länge Null zu, falls $\sigma_{i}$ keine Randzelle darstellt, d.h. $\partial \sigma_{i} \cap \partial \mathcal{D}=\emptyset$ gilt. Zudem verdeutlicht Bild 7 , dass $\mathbf{n}_{\sigma_{i}}^{k}$ den nach außen gerichteten Einheitsnormalenvektor am Zellrand $\ell_{\sigma_{i}}^{k}$ darstellt.

Ausgehend von der Evolutionsgleichung (9) untergliedert sich die weitere Vorgehensweise in zwei Schritte:

- Approximation der Rand- und Volumenintegrale.

- Diskretisierung des hieraus resultierenden Systems gewöhnlicher Differentialgleichungen.

Bei der Approximation der Integrale verwenden wir problemangepasste Ansätze und unterscheiden im Folgenden Verfahren für innere Kanten $\ell_{i j}^{k}$, Randkanten $\ell_{\sigma_{i}}^{k}$ und Zellen $\sigma_{i}$.

\subsection{Integration über innere Kanten}

Unter Verwendung des in Bild 6 dargestellten Mittelpunktes $x_{i j}^{k}$ der Kante $\ell_{i j}^{k}$ nutzen wir die einfache Mittelpunktregel gemäß

$$
\int_{\ell_{i j}^{k}} \lambda(x) \nabla_{x} T(x, t) \cdot \mathbf{n}_{i j}^{k} d s \approx\left|\ell_{i j}^{k}\right| \lambda\left(x_{i j}^{k}\right) \nabla_{x} T\left(x_{i j}^{k}, t\right) \cdot \mathbf{n}_{i j}^{k} .
$$

Da die Triangulierung so generiert wird, dass sich jedes Dreieck ausschließlich innerhalb einer Gewebeschicht befindet, ist die Auswertung des Wärmeleitkoeffizienten $\lambda$ direkt durch die Tabelle 2 festgelegt. Dagegen erfordert die 

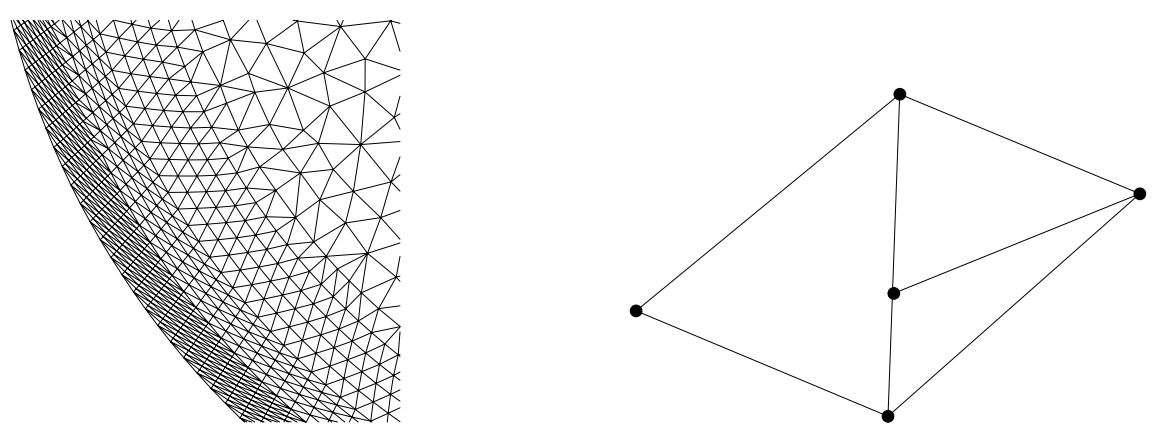

Abb. 4 Diskretisierung des Kopfbereichs (links), unzulässige Diskretisierung (rechts)

Berechnung des Temperaturgradienten eine genauere Betrachtung. Bedingt durch die gewählte Finite-Volumen-Formulierung stehen uns im Rahmen der numerischen Methode stets nur Zellmittelwerte $T_{i}(t)$ je Box $\sigma_{i}$ zur Verfügung. Zur Gradientenberechnung ordnen wir diesen Temperaturwert dem Punkt $x_{i}$ der Triangulierung zu, der im Fall eines inneren Knotens $x_{i} \notin \partial \mathcal{D}$ bei gleichseitigen Dreiecken dem Schwerpunkt der Zellen $\sigma_{i}$ entspricht. Betrachten wir das Dreieck $D$ mit den Eckpunkten

$$
x_{i}=\left(\begin{array}{c}
x_{i, 1} \\
x_{i, 2}
\end{array}\right), \quad x_{j}=\left(\begin{array}{c}
x_{j, 1} \\
x_{j, 2}
\end{array}\right), x_{m}=\left(\begin{array}{c}
x_{m, 1} \\
x_{m, 2}
\end{array}\right),
$$

so ergibt sich aus den zugehörigen Temperaturwerten $T_{i}, T_{j}$ und $T_{m}$ eine eindeutig bestimmte lineare Temperaturverteilung auf $D$. Der entsprechende Gradient im Punkt $x_{i j}^{k} \in D$ kann folglich in der Form

$$
\nabla_{x} T\left(x_{i j}^{k}, t\right)=\frac{1}{\operatorname{det}} \cdot\left(\begin{array}{cc}
x_{m, 2}-x_{i, 2} & x_{i, 2}-x_{j, 2} \\
x_{i, 1}-x_{m, 1} & x_{j, 1}-x_{i, 1}
\end{array}\right) \cdot\left(\begin{array}{c}
T_{j}(t)-T_{i}(t) \\
T_{m}(t)-T_{i}(t)
\end{array}\right)
$$

mit det $=\left(x_{j, 1}-x_{i, 1}\right)\left(x_{m, 2}-x_{i, 2}\right)-\left(x_{m, 1}-x_{i, 1}\right)\left(x_{j, 2}-x_{i, 2}\right)$ ermittelt werden, vergleiche [20]. Durch diese Vorgehensweise liegt mit

$$
\begin{aligned}
& \int_{\ell_{i j}^{k}} \lambda(x) \nabla_{x} T(x, t) \cdot \mathbf{n}_{i j}^{k} d s \\
& \approx \frac{\left|\ell_{i j}^{k}\right|}{\operatorname{det}} \lambda\left(x_{i j}^{k}\right)\left(\begin{array}{cc}
x_{m, 2}-x_{i, 2} & x_{i, 2}-x_{j, 2} \\
x_{i, 1}-x_{m, 1} & x_{j, 1}-x_{i, 1}
\end{array}\right) \cdot\left(\begin{array}{c}
T_{j}(t)-T_{i}(t) \\
T_{m}(t)-T_{i}(t)
\end{array}\right) \cdot \mathbf{n}_{i j}^{k}
\end{aligned}
$$

eine intuitiv verständliche, einfache und eindeutige Approximation der Integrale über innere Randkanten $\ell_{i j}^{k}$ vor, die zudem einen lokalen Charakter aufweist.

\subsection{Integration über Randkanten}

Die Auswertung der Integrale über die Geometrieränder $\ell_{\sigma_{i}}^{k} \subset \partial \mathcal{D}$ basiert auf einem ähnlichen Ansatz. Analog zur Berechnung der Wärmeflüsse über 


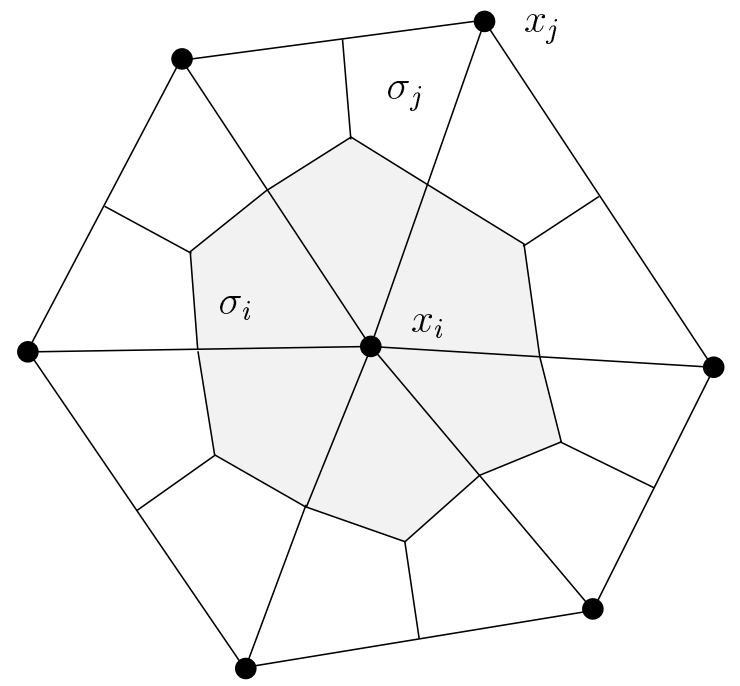

Abb. 5 Primäres und sekundäres Netz mit Zelle $\sigma_{i}$

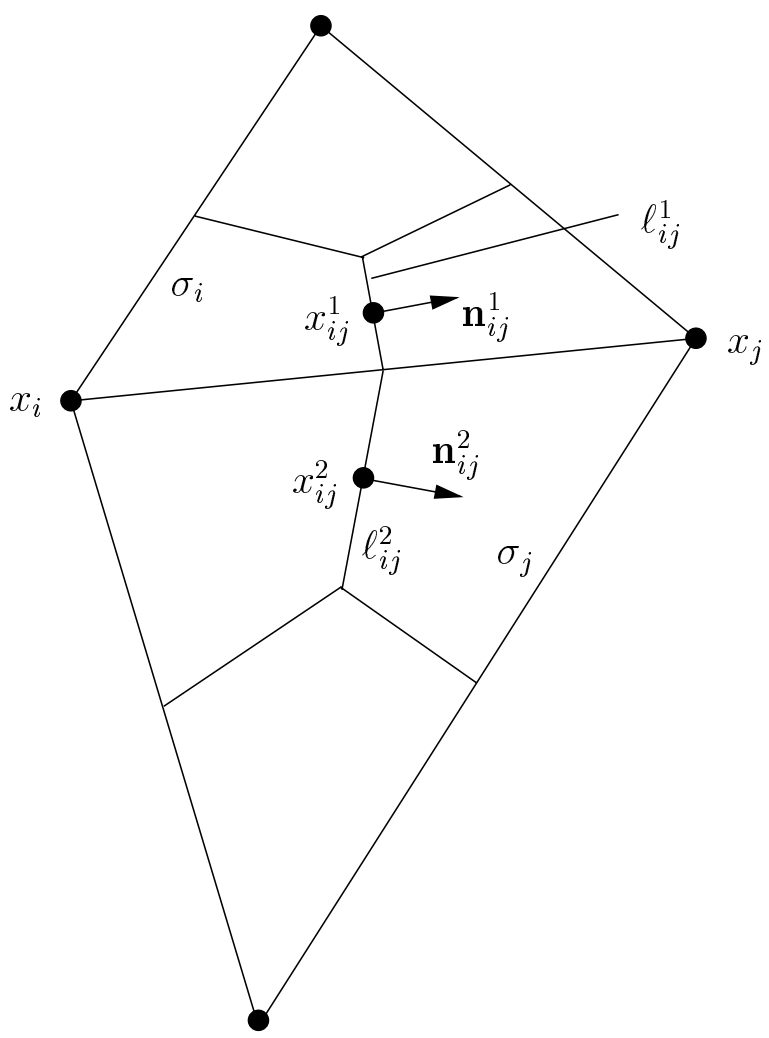

Abb. 6 Randkanten $\ell_{i j}^{k}, k=1,2$ zwischen den Zellen $\sigma_{i}$ und $\sigma_{j}$ 
innere Kanten $\ell_{i j}^{k}$ nutzen wir eine lineare Temperaturverteilung auf einem zugeordneten Dreieck $D$ zur Approximation des Temperaturgradienten am Mittelpunkt $x_{\sigma_{i}}^{k}$ des Geradenstücks $\ell_{\sigma_{i}}^{k}$. Im Gegensatz zu den inneren Zellrändern müssen an dieser Stelle zwei wesentliche Sachverhalte berücksichtigt werden.

Erstens ist es erforderlich, den in Abschnitt 2.1 angesprochenen DirichletRandbedingungen, d.h. den den Körperrandbereichen gemäß Abbildung 1 zugeordneten Randtemperaturen Rechnung zu tragen. Die an der Kante $\ell_{\sigma_{i}}^{k}$ laut Dirichlet-Randbedingung vorgegebene Außentemperatur $T_{\text {Rand }}$ ordnen wir daher sowohl dem Anfangspunkt $x_{i}$ als auch dem Endpunkt $x_{m}^{k}$ von $\ell_{\sigma_{i}}^{k}$ zu. Zweitens muß der Problematik entgegen getreten werden, dass der zur Randzelle $\sigma_{i}$ gehörige Knotenpunkt $x_{i}$ mit dem Anfangspunkt der Kante $\ell_{\sigma_{i}}^{k}$ übereinstimmt und folglich eine Lokalisierung des Zellmittelwertes $T_{i}$ am Punkt $x_{i}$ ausschließt.

Unter Verwendung der in Abbildung 7 genutzten Notation nutzen wir zur Lösung dieser Problemstellung den Knoten

$$
\widetilde{x}_{i}=x_{i}+\frac{\sqrt{3}}{8} \min \left\{\left\|x_{p}-x_{i}\right\|,\left\|x_{q}-x_{i}\right\|\right\} \cdot \mathbf{r},
$$

wobei $x_{p}$ und $x_{q}$ die verbleibenden zwei Eckpunktes des Dreiecks darstellen, das ausgehend von $x_{i}$ in Richtung $\mathbf{r}$ liegt. Der Richtungsvektor $\mathbf{r}$ ist hierbei durch

$$
\mathbf{r}=-\frac{\mathbf{n}_{\sigma_{i}}^{1}+\mathbf{n}_{\sigma_{i}}^{2}}{\left\|\mathbf{n}_{\sigma_{i}}^{1}+\mathbf{n}_{\sigma_{i}}^{2}\right\|}
$$

festgelegt. Die Berechnung ist dabei so gewählt, dass der Punkt $\widetilde{x}_{i}$ im Fall gleichseitiger Randdreiecke und paralleler Randkanten $\ell_{\sigma_{i}}^{1}$ und $\ell_{\sigma_{i}}^{2}$ dem Schwerpunkt der Box $\sigma_{i}$ entspricht. Desweiteren gilt für das durch die Punkte $x_{i}, x_{m}^{k} \in \ell_{\sigma_{i}}^{k}$ und $\widetilde{x}_{i}$ gebildeten Dreieck $D$ stets $D \subset \overline{\mathcal{D}}$, wodurch im Fall einer Differenz zwischen der Randtemperatur und dem Zellmittelwert $T_{i}$ stets die physikalisch adäquate Wärmeflußrichtung ermittelt wird. In [12] wurde nachgewiesen, dass durch die Verwendung des erweiterten Schwerpunktes $\widetilde{x}_{i}$ die resultierende Finite-Volumen-Methode einer diskreten Form des bekannten Minimum-Maximum-Prinzips [23,27] genügt.

Es sei hierbei bemerkt, dass die Nutzung des realen Schwerpunktes $x_{i}^{b}$ der Box $\sigma_{i}$ im Fall einer nicht konvexen Randzelle zu der Problematik $x_{i}^{b} \notin \overline{\mathcal{D}}$ und folglich zu unphysikalischen Randflüssen führen kann, die eine Verletzung des Minimum-Maximum-Prinzips nach sich zieht. Entsprechend der Vorgehensweise (10) erhalten wir unter Verwendung der obigen Punkte $\widetilde{x}_{i}, x_{i}$ und $x_{m}^{k}$ den Gradienten am Randpunkt $x_{\sigma_{i}}^{k}$ gemäß

$$
\nabla_{x} T\left(x_{\sigma_{i}}^{k}, t\right)=\frac{1}{\operatorname{det}} \cdot\left(\begin{array}{cc}
x_{m, 2}^{k}-\widetilde{x}_{i, 2} & \widetilde{x}_{i, 2}-x_{i, 2} \\
\widetilde{x}_{i, 1}-x_{m, 1}^{k} & x_{i, 1}-\widetilde{x}_{i, 1}
\end{array}\right) \cdot\left(\begin{array}{l}
T_{\text {Rand }}(t)-T_{i}(t) \\
T_{\text {Rand }}(t)-T_{i}(t)
\end{array}\right)
$$

mit det $=\left(x_{i, 1}-\widetilde{x}_{i, 1}\right)\left(x_{m, 2}^{k}-\widetilde{x}_{i, 2}\right)-\left(x_{m, 1}^{k}-\widetilde{x}_{i, 1}\right)\left(x_{i, 2}-\widetilde{x}_{i, 2}\right)$, und hiermit, unter Benutzung der Abkürzung

$$
\kappa_{i}:=\frac{\left|\ell_{\sigma_{i}}^{k}\right|}{\operatorname{det}} \lambda\left(x_{\sigma_{i}}^{k}\right)
$$




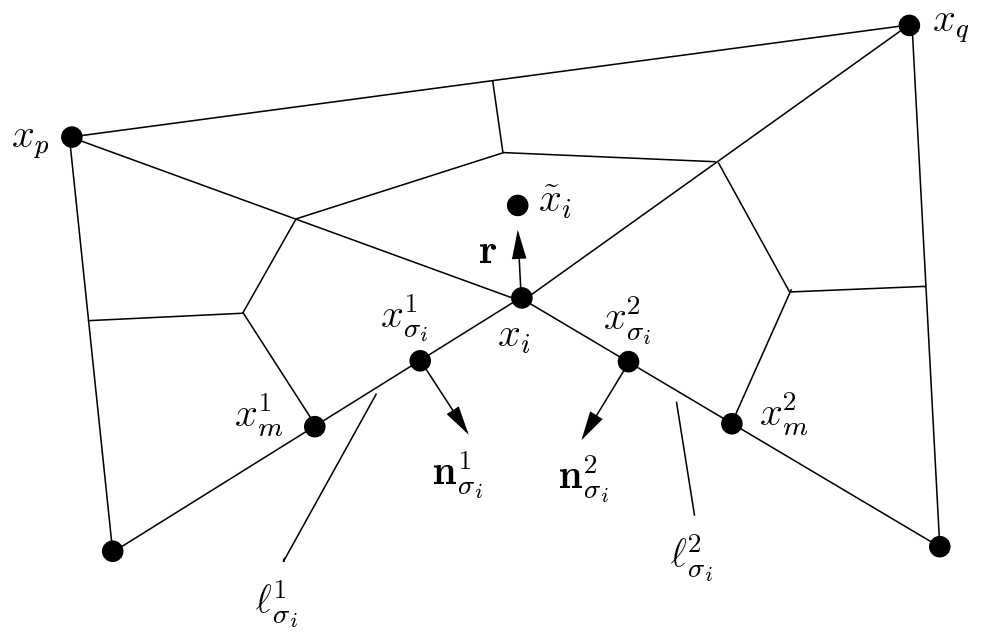

Abb. 7 Notation bei Randzellen $\sigma_{i}$

die Approximation

$$
\begin{aligned}
& \int_{\ell_{\sigma_{i}}^{k}} \lambda(x) \nabla_{x} T(x, t) \cdot \mathbf{n}_{\sigma_{i}}^{k} d s \\
& \approx \kappa_{i}\left(\begin{array}{ll}
x_{m, 2}-\widetilde{x}_{i, 2} & \widetilde{x}_{i, 2}-x_{j, 2} \\
\widetilde{x}_{i, 1}-x_{m, 1} & x_{j, 1}-\widetilde{x}_{i, 1}
\end{array}\right) \cdot\left(\begin{array}{l}
T_{R a n d}(t)-T_{i}(t) \\
T_{R a n d}(t)-T_{i}(t)
\end{array}\right) \cdot \mathbf{n}_{\sigma_{i}}^{k} .
\end{aligned}
$$

\subsection{Integration über Zellen}

Die Berechnung der Quellen- bzw. Senkenterme erfolgt direkt durch Auswertung der jeweiligen Funktion am Knotenpunkt $x_{i}$ der Zelle $\sigma_{i}$. Für die metabolische Wärmeproduktion erhalten wir hiermit

$$
\int_{\sigma_{i}} Q_{M}(x) d x \approx\left|\sigma_{i}\right| Q_{M}\left(x_{i}\right),
$$

wobei der explizite Wert der Quellfunktion $Q_{M}$ entsprechend des vorliegenden Körperkompartimentes und der Gewebeart gemäß Tabelle 3 festgelegt ist.

Im Hinblick auf den Blutfluß wird die Temperatur des zentralen Blutreservoirs durch

$$
\begin{aligned}
T_{B L}(t) & =\frac{\int_{\mathcal{D}} K(x) B F(x) T(x, t) d x}{\int_{\mathcal{D}} K(x) B F(x) d x} \\
& \approx \frac{\sum_{i=1}^{n} K\left(x_{i}\right) B F\left(x_{i}\right) T_{i}(t)}{\sum_{i=1}^{n} K\left(x_{i}\right) B F\left(x_{i}\right)}
\end{aligned}
$$


berechnet. Einsetzen dieses zeitabhängigen Wertes in die Darstellung (4) und Integration über die Zelle $\sigma_{i}$ liefert

$$
\int_{\sigma_{i}} Q_{B}(x, t) d x \approx\left|\sigma_{i}\right| \rho_{B L} \cdot c_{B L} \cdot K\left(x_{i}\right) B F\left(x_{i}\right)\left(T_{B L}(t)-T_{i}(t)\right) .
$$

Im Gegenteil zu den Randintegralen liefert die Approximation des Blutflußmodells durch (14) eine globale Kopplung aller Temperaturwerte.

\subsection{Zeitintegration}

Nutzung der numerischen Quadraturformeln (10), (12), (13) und (15) führt auf die sogenannte semidiskrete Form der Evolutionsgleichung (7), die ein System gewöhnlicher Differentialgleichungen für die Zellmittelwerte der Temperatur in der Form

$$
\frac{d}{d t} T_{i}(t)=F_{i}(t)+Q_{i}(t), \quad i=1, \ldots, n
$$

darstellt. Hierbei wurden in $F_{i}(t)$ alle numerischen Quadraturformeln der Randintegrale und in $Q_{i}(t)$ alle numerischen Quadraturformeln der Volumenintegrale subsummiert, d.h. $F_{i}$ beinhaltet aufgrund der lokalen Abhängigkeit der Gradientenauswertung am Rand des Kontrollvolumens ausschließlich Temperaturzellmittelwerte $T_{j}(t)$ mit $j \in \mathcal{N}(i) \cup\{i\}$, während $Q_{i}(t)$, bedingt durch den Quellterm (15), eine globale Abhängigkeit von den Zellmittelwerten der Temperatur über alle Kontrollvolumina aufweist. Bei Vernachlässigung der kompensierenden Wirkung des Blutflusses liegt für den Zellmittelwert $T_{i}(t)$ ausschließlich eine Abhängigkeit von den Temperaturwerten der Box $\sigma_{i}$ und den Nachbarzellen $\sigma_{j}$ mit $j \in \mathcal{N}(i)$ über die Randintegralapproximationen (10) und (12) vor.

Zur Diskretisierung derartiger gekoppelter Gleichungen stehen heute eine Vielzahl unterschiedlicher Zeitschrittverfahren zur Verfügung, die üblicherweise in Ein- und Mehrschrittverfahren gruppiert werden. Basierend auf einer Unterteilung der Zeit $t^{n+1}=t^{n}+\Delta t^{n}$ mit $t^{0}=0$ und $\Delta t^{n}>0$ wird eine Approximation der Zeitableitung unter Berücksichtigung der Anfangsbedingungen

$$
T_{i}\left(t^{0}\right)=\frac{1}{\left|\sigma_{i}\right|} \int_{\sigma_{i}} T\left(x, t^{0}\right) d x
$$

vorgenommen. Verwendet man zur Ermittlung der Zellmittelwerte $T_{i}\left(t^{n+1}\right)$ ausschließlich Werte der Zeitschicht $t^{n}$, so sprechen wir von einem Einschrittverfahren. Zu dieser Klasse gehören alle bekannten Runge-Kutta-Verfahren wie auch das klassische Euler-Verfahren und die Trapezregel. Werden zudem noch Werte früherer Zeitebenen in die Berechnung der Temperaturverteilung zum Zeitpunkt $t^{n+1}$ herangezogen, so erhalten wir ein Mehrschrittverfahren. Typische Vertreter dieser Gruppe sind die Adams-Typ-Methoden wie auch das Milne-Simpson- und das Nyström-Verfahren, die sich sämtlichst aus einer Integration der Differentialgleichung und anschließender Anwendung einer interpolatorischen Quadraturformel ergeben. Die Approximation der in (16) 
auftretenden Ableitung durch einen Differenzenquotienten führt auf die bekannten BDF-Verfahren (backward differential formulas), die im Vergleich zu den zuvorgenannten Mehrschrittverfahren über einen zumeist deutlich größeren Stabilitätsbereich verfügen und folglich besser für steife Differentialgleichungen geeignet sind. Hinsichtlich einer ausführlichen Herleitung und Diskussion einer großen Bandbreite numerischer Zeitschrittverfahren sei an dieser Stelle auf die sehr gut lesbaren Lehrbücher von Hanke-Bourgeois [14], Schwarz/Köckler [24] und Plato [22] verwiesen.

Blicken wir kurz auf den Anfang des Kapitels 3 zurück. Durch die Einschränkung auf stationäre Zustände und der hierbei genutzten Festlegung $\rho(x) c(x) \equiv 1$ besitzt erst der stationäre Endzustand mit $\frac{\partial T}{\partial t}(x, t)=0$ physikalische Relevanz. Die zeitliche Evolution kann folglich als Iteration zum stationären Zustand interpretiert werden, wobei die erzielten Zwischenzustände keine thermodynamische Bedeutung aufweisen. Folglich können wir uns bei der Zeitdiskretisierung auf sehr einfache Algorithmen beziehen, da die Genauigkeit der gewählten Approximation nicht von Belang ist. Wir nutzen daher das klassiche explizite Euler-Verfahren, das auch als Eulersche Polygonzugmethode bekannt ist. Hierbei betrachten wir das System gewöhnlicher Differentialgleichungen (16) zum Zeitpunkt $t^{n}$ und ersetzen die auftretende Ableitung durch einen einfachen Differenzenquotienten der Form

$$
\frac{d}{d t} T_{i}\left(t^{n}\right)=\frac{T_{i}\left(t^{n+1}\right)-T_{i}\left(t^{n}\right)}{\Delta t^{n}}
$$

so dass das numerische Verfahren die Darstellung

$$
T_{i}\left(t^{n+1}\right)=T_{i}\left(t^{n}\right)+\Delta t^{n}\left(F_{i}\left(t^{n}\right)+Q_{i}\left(t^{n}\right)\right), \quad i=1, \ldots, n
$$

erhält. Die Nutzung eines expliziten Zeitintegrationsverfahren zieht jedoch aus Stabilitätsgründen den Nachteil einer oftmals sehr drastischen Zeitschrittweitenrestriktion nach sich. In dem vorliegenden Fall ergibt sich

$$
\Delta t^{n} \leq \min _{i=1, \ldots, n} \frac{\left(\min _{x \in \partial \sigma_{i}}\left\|x-\widetilde{x}_{i}\right\|\right)^{2}}{\lambda\left(\widetilde{x}_{i}\right)}
$$

wobei $\widetilde{x}_{i}$ den Schwerpunkt bzw. bei Randzellen den erweiterten Schwerpunkt darstellt. Hierdurch wird deutlich, dass die im Bereich des Kopfes vorgenommene feine Diskretisierung der Körperrandschichten eine extrem kleine zulässige Zeitschrittweite bedingt. Derartige Einschränkungen können durch eine implizite Zeitintegration vermieden werden. Eine mögliche Vorgehensweise ergibt sich in der Form

$$
T_{i}\left(t^{n+1}\right)=T_{i}\left(t^{n}\right)+\Delta t^{n}\left(F_{i}\left(t^{n+1}\right)+Q_{i}\left(t^{n+1}\right)\right), \quad i=1, \ldots, n .
$$

Die involvierte Auswertung der rechten Seite zum Zeitpunkt $t^{n+1}$ führt auf jeweils ein lineares Gleichungssystem, das in jedem Zeitschritt zu lösen ist. Die von der aktuellen Zeitschrittweite $\Delta t^{n}$ abhängige Matrix ist dabei aufgrund der in dem Quellterm beinhalteten globalen Abhängigkeit voll besetzt, d. h., dass alle Matrixelemente von Null verschiedene Einträge aufweisen können. Hierdurch liegt einerseits ein sehr hoher Speicherplatzbedarf vor und andererseits ergibt sich bei der Nutzung iterativer Gleichungssystemlöser 
die Problematik, dass Matrix-Vektor-Multiplikationen einen Aufwand der Größenordnung $\mathcal{O}\left(n^{2}\right)$ aufweisen. In [12] wird daher die Nutzung einer semiimpliziten Variante gemäß

$$
T_{i}\left(t^{n+1}\right)=T_{i}\left(t^{n}\right)+\Delta t^{n}\left(F_{i}\left(t^{n+1}\right)+Q_{i}\left(t^{n}\right)\right), \quad i=1, \ldots, n .
$$

vorgeschlagen, wodurch eine signifikante Beschleunigung des Verfahrens erzielt werden konnte. Der Grund für diesen positiven Effekt liegt bei dem erwähnten Ansatz zum einen in der Nutzbarkeit großer Zeitschrittweiten $\Delta t^{n}$ und zum anderen in der Eigenschaft, dass bei der auftretenden Matrix innerhalb jeder Zeile $j$ alle Einträge $a_{j k}$ mit $k \notin \mathcal{N}(j) \cup\{j\}$ identisch verschwinden. Durch letzteres wird der Speicherplatzbedarf deutlich gesenkt und der Aufwand pro Matrix-Vektor-Multiplikation auf die Größenordnung $\mathcal{O}(n)$ verringert. Zur iterativen Lösung dieser Gleichungssysteme mit einer derartigen großen und schwach besetzten Matrix stehen heutzutage sehr effiziente präkonditionierte Krylov-Unterraum-Verfahren zur Verfügung. Eine Einführung in klassische und moderne Algorithmen zur schnellen Lösung linearer Gleichungssysteme findet der interessierte Leser in [19].

\section{Anwendungsbeispiele}

Das Verfahren wird im Weiteren anhand zweier praxisrelevanter Problemstellungen untersucht. Ein besonderes Augenmerk soll hierbei einerseits auf die Einhaltung des Minimum-Maximum-Prinzips und andererseits auf die Wirkung der Quellterme für die metabolische Wärmeproduktion und den Blutfluß gelegt werden.

Für die numerischen Simulationen nutzen wir die in Abbildung 3 dargestellte Triangulierung, die 37351 Dreiecke und 19092 Knotenpunkte aufweist. Entsprechend der Definition der Kontrolvolumina ist jedem Knotenpunkt genau eine Zelle zugeordnet, wodurch die Anzahl der Punkte stets mit der Anzahl der Zellen übereinstimmt.

Zunächst wenden wir uns einer üblichen Situation innerhalb eines Inkubators zu. Die Umgebungstemperatur wird mit $34,5^{\circ} \mathrm{C}=308,65 \mathrm{~K}$ auf einem konstanten Niveau gehalten, während durch die Wärmematte im Rückenbereich eine auf $37^{\circ} \mathrm{C}=310,15 \mathrm{~K}$ erhöhte Randtemperatur vorliegt. Die bei diesen Randbedingungen und einer Anfangstemperaturverteilung von $37^{\circ} \mathrm{C}=310,15 \mathrm{~K}$ für den stationären Endzustand erzielten numerischen Resultate sind in der Abbildung 8 aufgeführt, wobei die Farbcodierung und Isolinienverteilung entsprechend des aufgeführten Farbbalkens verwendet wurden. Die obere Darstellung innerhalb der Abbildung 8 spiegelt die Temperaturverteilung bei gegebenen Anfangs- und Randbedingungen unter Vernachlässigung beider Quellterme wieder. Die Isolinien verlaufen hierbei stets von einem Randbereich zu einem anderen Randstück. Es treten keine geschlossenen Linienzüge auf, wodurch sehr schön die Einhaltung des MinimumMaximum-Prinzips verdeutlicht wird, da sowohl das Temperaturminimum als auch das Temperaturmaximum am Rand des Körpers angenommen werden. Im mittleren Bild ist das Ergebnis unter Berücksichtigung der metabolischen Wärmeproduktion aufgeführt. Sehr eindrucksvoll ist hierbei die drastische Temperaturerhöhung im Kopfbereich zu erkennen. Ein weiteres lokales 


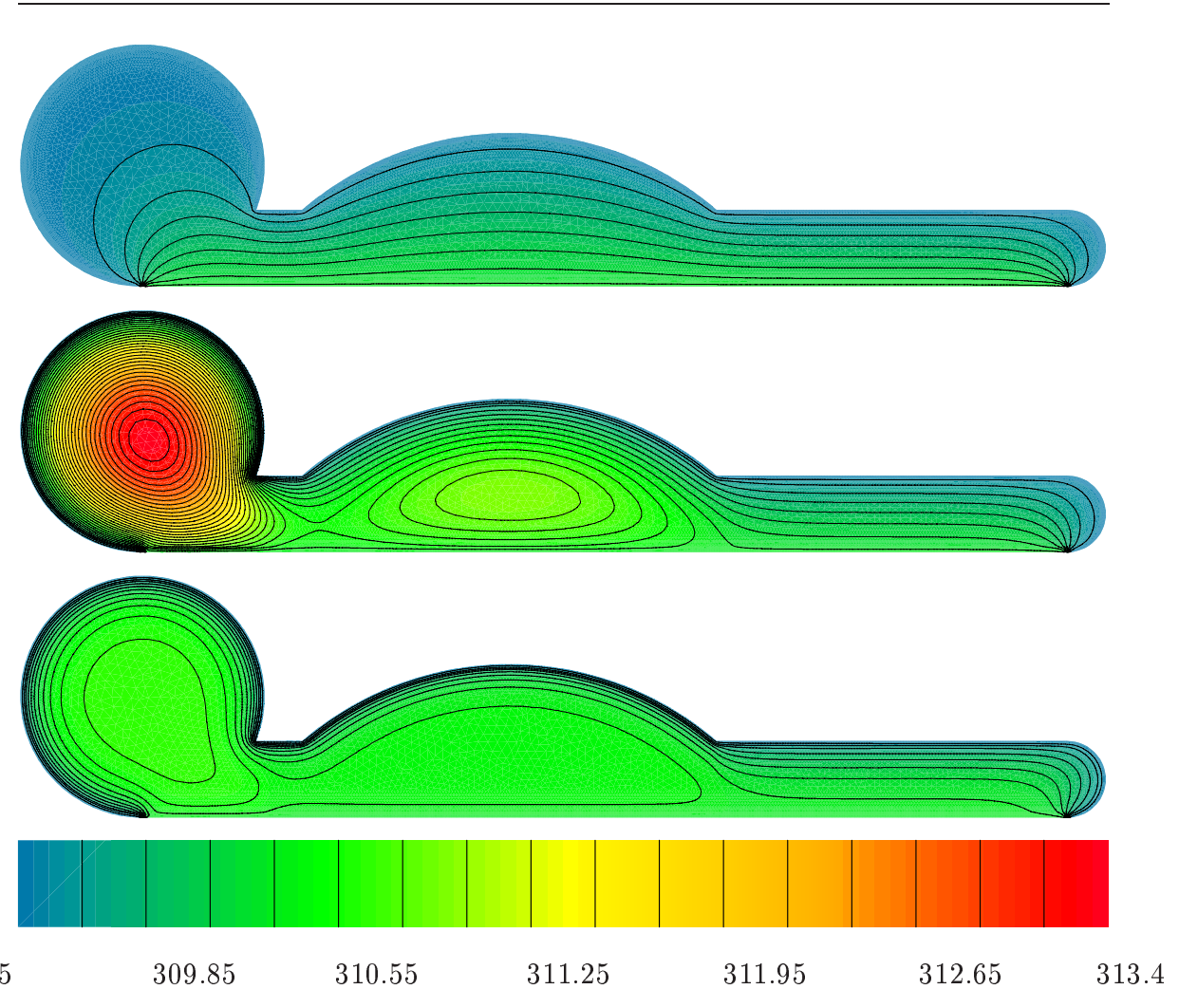

Abb. 8 Numerische Resultate zur stationären Temperaturverteilung der Wärmeleitungsgleichung (oben) mit metabolischer Wärmeproduktion (Mitte) und zusätzlichem Einfluß der Blutzirkulation (unten).

Maximum tritt zudem im Bereich des Bauches auf. Beide Extrema der Temperatur korrelieren sehr gut mit den im Modell festgelegten metabolischen Wärmeproduktionswerten gemäß Tabelle 3. Abschliessend wird im dritten Bild die Temperaturverteilung unter Berücksichtigung beider Quellterme verdeutlicht. Die wärmeverteilende Wirkung des Blutflußmodells wird bei dieser Darstellung hervorragend sichtbar. Die Maxima sind deutlich reduziert worden und es hat ein offensichtlicher Wärmeausgleich stattgefunden. Bereits innerhalb dieser Grafik sind erhöhte Temperaturgradienten in den Kopfrandbereichen gut erkennbar.

Mit dem zweiten Anwendungsbeispiel untersuchen wir die eingangs aufgeworfene Fragestellung einer lokalen Kühlung des Gehirnbereichs durch Absenkung der Kopfrandtemperatur. Die Anfangstemperaturverteilung wie auch die Randtemperaturen wurden mit Ausnahme der Kopfrandtemperatur identisch zur ersten Problemstellung gewählt. Bei der Bedingung am Rand des Kopfbereiches wurde hingegen eine Absenkung der Temperatur auf $26^{\circ} \mathrm{C}=$ $299,15 K$ vorgenommen. Bei Vernachlässigung der metabolischen Wärmeproduktion und des Blutflusses können wir der oberen Darstellung in Abbildung 


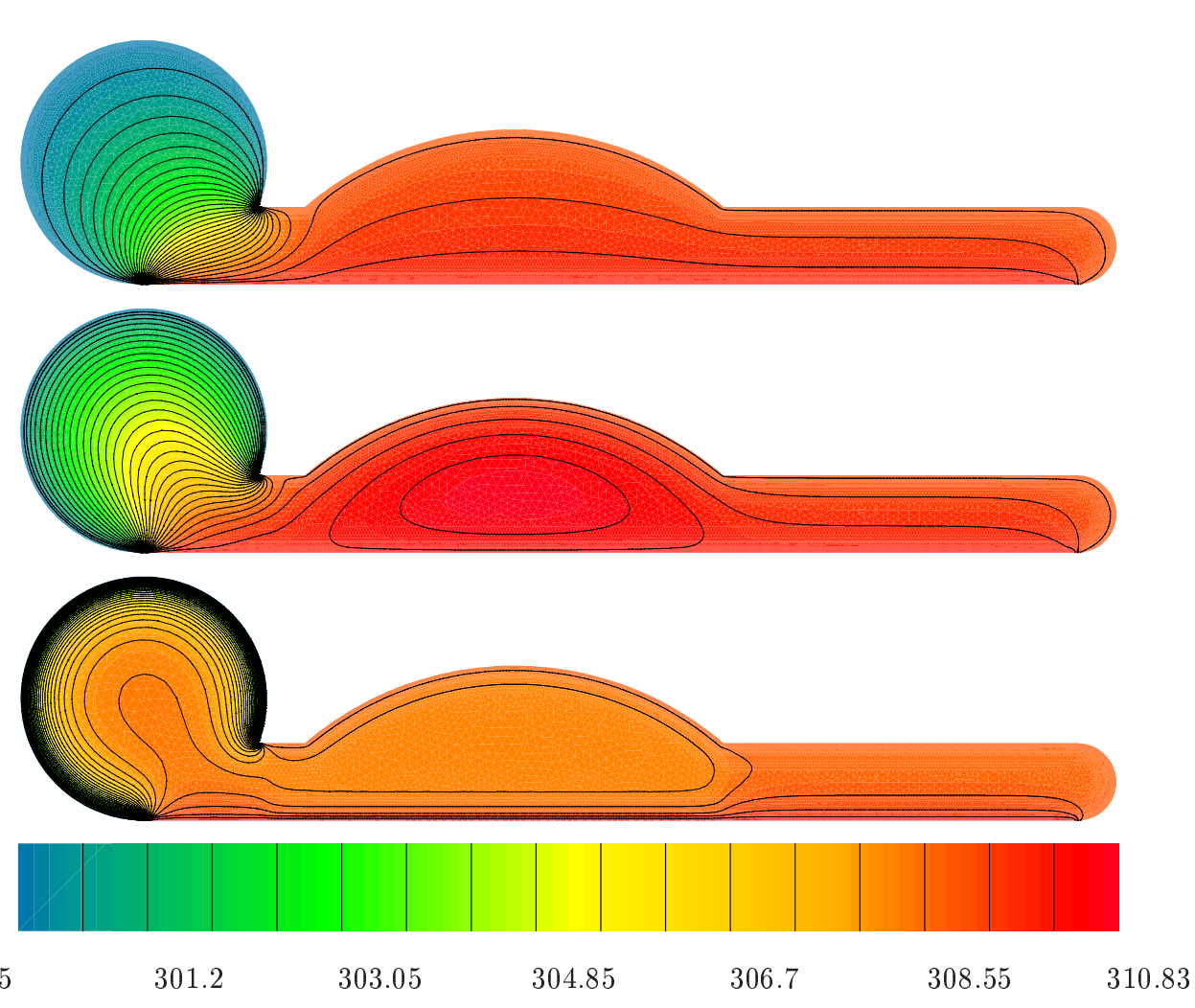

Abb. 9 Numerische Resultate zur stationären Temperaturverteilung der Wärmeleitungsgleichung (oben) mit metabolischer Wärmeproduktion (Mitte) und zusätzlichem Einfluß der Blutzirkulation (unten).

9 die zu erwartende Absenkung innerhalb des gesamten Kopfbereiches entnehmen. Analog zum ersten Testfall liegen keine geschlossenen Isolinien vor, da auch bei diesem Anwendungsfall das Minimum-Maximum-Prinzip eingehalten wurde. Durch die Einbindung der metabolischen Wärmeproduktion zeigt sich auch in diesem Fall eine erste Erhöhung der Kopftemperatur aufgrund der starken lokalen Wärmezufuhr in diesem Bereich. Eindrucksvoll wird im dritten Bild die Wärmeverteilung aufgrund des Blutflusses deutlich. Die angelegte Randtemperatur beeinflusst im wesentlichen die äußersten Kopfrandschichten und weist eine vergleichsweise geringe Wirkung im inneren Gehirnbereich auf; während die im Bereich des Gehirns geforderte Kühlung des Gewebes um $2-3 K$ erfolgt ist, zeigt sich im Kern des Torsos eine Unterkühlung. Nach der im Kontext der vorliegenden Modellierung durchgeführten numerischen Simulation erweist sich eine Reduktion der Gehirntemperatur auf der Grundlage einer lokalen Absenkung der Umgebungstemperatur im Kopfbereich daher als nicht praktikabel. 
Danksagung Die Autoren möchten sich an dieser Stelle herzlich bei Dr. Jochim Koch, Abteilung für Grundlagenentwicklung der Dräger AG Lübeck, für die konstruktive Zusammenarbeit bei der Modellierung bedanken. Desweiteren gilt unser Dank Herrn Dr. Oliver Friedrich für die Bereitstellung des Gittergenerators und des Visualisierungstools, Herrn Dipl.-Math. Martin Ludwig für die Umsetzung einiger Programmodule sowie Herrn K. Strube für die präzise Anfertigung einiger Grafiken.

\section{Literatur}

1. Breuß, M., Dolejší, V. und Meister, A.: Anisotropic Adaptive Resolution of Boundary Layers for Heat Conduction Problems. Preprint 03/05, Fachbereich für Mathematik und Informatik, Universität Kassel.

2. Breuß, M., Fischer, B. und Meister, A.: The unsteady thermoregulation of premature infants - a model and its application. In: T. Sonar and I. Thomas (eds.) Proceedings of the GAMM-Workshop: Discrete Modelling and Discrete Algorithms in Continuum Mechanics, Logos, Berlin, 47-56 (2001)

3. Breuß, M., Fischer, B. und Meister, A.: The Numerical Simulation of Unsteady Heat Conduction in a Premature Infant. Int. J. Num. Meth. Fluids 40, 253-261 (2002)

4. Breuß, M., Fischer, B. und Meister, A.: An application of a blood flow model. In: J. Levesley, I. Anderson, J.C. Mason (eds.) Proceedings of the International Symposium on Algorithms for Approximation IV, Huddersfield, 428-436 (2002)

5. Brück, K.: Temperature regulation in the newborn infant. Biol. Neonate 3, 65119 (1961)

6. Brück, K.: Heat Production and Temperature Regulation. In: U. Stave (ed.) Physiology of the neonatal period, Appleton-Century-Crofts, 493-557 (1970)

7. Bußmann, O.: Modell der Thermoregulation des Früh- und Neugeborenen unter Einbeziehung der thermischen Reife. Dissertation, Institut für Medizintechnik, MU Lübeck (2000)

8. Busto, R. et al.: The importance of brain temperature in cerebral ischemic injury. Stroke 20, 1114-1134 (1989)

9. Duck, F. A.: Physical Properties of tissue - 1. mammals. Academic Press, London (1990)

10. Fiala, D., Lomas, K. J. und Stohrer, M.: A computer model of human thermoregulation for a wide range of environmental conditions: the passive system. J. Appl. Physiol. 87(5), 1957-1999 (1999)

11. Fischer, B., Ludwig, M. und Meister, A.: A finite volume method to compute the steady state temperature distribution in premature or newborn infants. ZAMM 81, 759-760 (2001).

12. Fischer, B., Ludwig, M. und Meister, A.: The Thermoregulation of Infants: Modeling and Numerical Simulation. BIT 41, Nr. 5, 950-966 (2001)

13. Gluckman, P.D. und Williams, C.E.: When and why do brain cells die? Dev. Med. Child Neurol. 34, 1010-1014 (1992)

14. Hanke-Bourgeois, M.: Grundlagen der Numerische Mathematik und des Wissenschaftlichen Rechnens, Teubner (2002)

15. Holliday, M.A.: Metabolic rate and organ size during growth from infancy to maturity and during late gestation and early infancy. Pediatrics $47(1)$, suppl. 2, $169+(1971)$

16. Loziichuk, N. G. und Onopchuk, Yu. N.: Mathematical Models of the Thermoregulation System of the Organism and their Analysis. Cybernetics and Systems Analysis 31, Nr. 4, 605-617 (1995)

17. Ludwig, M.: Die numerische Simulation der Temperaturverteilungen in Frühund Neugeborenen. Diplomarbeit, FB Mathematik, Universität Hamburg (1999)

18. Mallard, E.C. et al.: Neuronal damage in the developing brain following intrauterine asphyxia. Reprod. Fertil. Dev. 7, 647-653 (1995)

19. Meister, A.: Numerik linearer Gleichungssysteme, 2. Auflage, Vieweg (2005)

20. Meister, A. und Sonar, T.: Finite-volume schemes for compressible fluid flow. Surv. Math. Ind. 8, 1-36 (1998) 
21. Pennes, H.H.: Analysis of tissue and arterial blood temperatures in the resting human forearm. J. Appl. Physiol. 1, 93-122 (1948)

22. Plato, R.: Numerische Mathematik kompakt, 2. Auflage, Vieweg (2004)

23. Protter, M. H. und Weinberger, H. F.: Maximum principles in differential equations. Prentice-Hall, New Jersey (1967)

24. Schwarz, H. R. und Köckler, N.: Numerische Mathematik, 5. Auflage, Teubner (2004)

25. Simbruner, G.: Thermodynamic Models for Diagnostic Purposes in the Newborn and Fetus. Facultas Verlag, Wien (1983)

26. Thomas, K.: Thermoregulation in neonates. Neonatal Network 13(2), 15-22 (1994)

27. Tveito, A. und Winther, R.: Einführung in partielle Differentialgleichungen. Springer Verlag, New York, Berlin, Heidelberg, (2002)

28. Werner, J.: Regelung der menschlichen Körpertemperatur. de Gruyter (1984)

29. Werner, J.: Thermoregulatory models. Scand. J. Work Environ. Health 15, suppl. 1, 34-46 (1989)

30. Witzleb, E.: Funktionen des Gefäßsystems. In: R. Schmidt und G. Tews (eds.) Physiologie des Menschen, Springer, 505-572 (1990) 